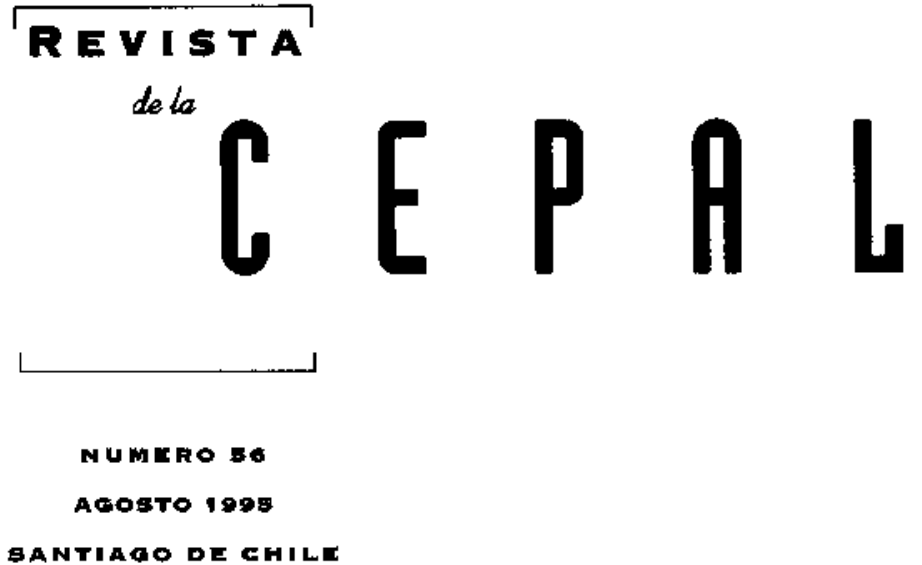

ANIBAL PINTO

Director

EUGENIO LAHERA

Secretario Técnico

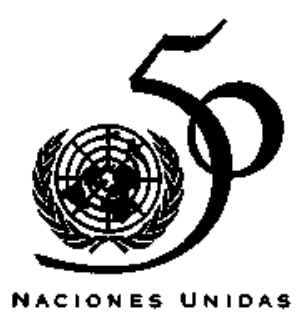


Democracla y desarrollo

Fernando H. Cardoso

¿Es posible crecer con equidad?

Joseph Ramos

Estabilidad y estructura: Interacclones en el crecimiento económlco

Jose Maria Fanelli y Roberto Frenkel

Reforma a los sistemas de pensiones en América Latina

Andras Uthoff

Tendenclas económicas en China: significado para el comerclo con América Latina y el Caribe

Mikio Kuwayama

El Intercamblo económico entre América Latina y las

economias dinámicas de Asla

Ronald Sprout

La relación económica entre la Amórica Latina y la Unlón Europea

Roberto Smith Perera

Nuevas implicaclones de las reglas do orlgen

Eduardo Gitli

Globalización y reestructuración energética en Amórlca Latina

Fernando Sánchez Albavera

El caloidoscoplo de la competitlvidad

Geraldo Muller

La privatización de los servicios públicos del agua

Miguel Solanes

¿Cuénto se puede gastar en educaclón?

Guillermo Labarca

Mujeres y migranteo: deslgualdades en el

mercado laboral de Santlago de Chile

Ivonne Szasz

Orlentaciones para los colaboradores de la Rovista de la CEPAL 


\section{Estabilidad y estructura: interacciones en el crecimiento económico}

\section{José María Fanelli} Roberto Frenkel

Investigador titular del CEDES $y$ del CONICET, y Director de La Carrera de Economfa de la Universidad de Buenos Aires.

Director del Area de Economí, CEDEs, Buenos Aires.
La evolución economica reciente de la región confirma que en ella la consolidación de la estabilidad no es todavía un hecho. Persisten algunos factores estructurales de inestabilidad macroeconómica, aún no han sido superadas diversas secuelas de la crisis de la deuda y han aparecido nuevas tensiones macroeconomicas de diverso origen. El desafío actual de la politica economica es el de llevar a cabo políticas de reforma y crecimiento, conservando los logros recientes en términos de estabilidad. Como requisito previo, debe haber una adecuada comprensión de la forma en que interactúan los factores macroeconómicos y microeconomicos; cómo se condicionan mutuamente las restricciones de consistencia macroeconomica, por un lado, y los desequilibrios generados durante el proceso de reformas y los cambios consecuentes en la base productiva, por otro. En este artículo se analiza por qué "el problema macroeconomico" tiene entidad propia y cómo afecta a la estructura microeconómica, tanto real como financiera, y es afectado por ella. En seguida se hace un intento por identificar los canales de transmisión micro-macro de mayor relevancia, y por último, se formulan algunas reflexiones sobre la forma en que las interacciones micro-macro contribuyen a determinar la potencialidad de crecimiento de una economía. 
I

\section{Introducción}

Durante los años ochenta, la crisis de la deuda generó un nivel inédito de inestabilidad macroeconómica. En ese contexto, la estabilización adquirió una prioridad absoluta y la cuestión del crecimiento fue relegada a un segundo plano. En los últimos años, sin embargo, la problemática relacionada con el crecimiento y el desarrollo productivo ha ido ganando espacio en la agenda de política económica en América Latina. Esto, sin lugar a dudas, representa un importante avance.

El factor fundamental que posibilitó el cambio en las prioridades asignadas a la estabilidad y el crecimiento ha sido la transformación operada en el mercado internacional de capitales: en lo que va de los años noventa se registró una fuerte caída en las tasas de interés externas y se flexibilizó apreciablemente el racionamiento del crédito que los países de la región venían sufriendo. Se desactivaron así los dos factores fundamentales que habian actuado como generadores de la crisis de la deuda. Bajo estas nuevas circunstancias, los gobiernos han contado con más libertad para llevar a cabo en forma exitosa políticas de estabilizacion y, como consecuencia, se observa una generalizada mejoría de las variables agregadas claves en los países de la región, con la excepción importante de Brasil (Damill, Fanelli, Frenkel y Rozenwurcel, 1993). Al dejar de ser la estabilización una necesidad tan acuciante, se abrí la posibilidad de pensar en el crecimiento. Como regla general, se ha estado buscando reactivar las fuentes de éste a través de reformas estructurales orientadas a profundizar el rol de los mercados y a aumentar la eficiencia de la estructura productiva.

Debe tenerse en cuenta, no obstante, que la consolidación de la estabilidad está lejos de ser un hecho. Algunos factores estructurales de inestabilidad macroeconómica están intactos, la crisis de la deuda dejo secuelas que no han sido superadas y han aparecido nuevas tensiones macroeconómicas como subproducto de la aceleración del ritmo de implementación de las reformas estructurales.

$\square$ Este artículo es una versión abreviada del texto "Estabilidad y estructura: interacciones en el crecimiento económico" preparado para la CEPAL, Santiago de Chile, 1994. Los autores agradecen el apoyo de la CEPAL, el International Development Research Center (IDRC) de Canada, la Swedish Agency for Research Cooperation with Developing Countries (SAREC) de Suecia, el North-South Center (Universidad de Miami) y la Mellon Foundation (Estados Unidos).
Hemos planteado que la nueva situación representa un avance. Pero si se toman en cuenta los factores potenciales de desequilibrio remanentes se hace necesario agregar que también representa un desafío, en esencia, el de cómo utilizar inteligentemente el mayor espacio para poner en marcha políticas de reforma y crecimiento, conservando los logros recientes en términos de estabilidad. Una condición para enfrentar este desafío es entender -aunque sólo sea de manera rudimentaria- la forma en que interactúan los factores macroeconómicos y microeconómicos. Esto es, entender cómo las restricciones de consistencia macroeconómica (de corto y largo plazo) condicionan los desequilibrios generados durante el proceso de reformas y por los cambios consecuentes en la base productiva, y a la vez son condicionadas por ellos.

Este artículo analiza los factores que afectan la relación entre consistencia agregada y desarrollo productivo. Para tratar esta problemática es ineludible utilizar resultados analíticos pertenecientes no sólo a la macroeconomfa y la microeconomía sino también a la teoría del crecimiento, la teoría financiera, las finanzas públicas, la organización industrial, el comercio internacional y la dinámica del cambio tecnologico. El enfoque ha de ser necesariamente interdisciplinario para ser fructífero. Este es, precisamente, uno de los obstáculos más difíciles de superar. La interacción sistemática en el plano analítico entre los macroeconomistas y los especialistas pertenecientes a cada una de las ramas mencionadas -en particular aquellos dedicados al análisis de la organización industrial, la innovación y el cambio tecnológico- es reducida. Es por ello que este artículo no puede sino ser de carácter exploratorio (entre otras cosas, debido al sesgo macroeconómico de la formación de sus autores). Nuestro mayor anhelo al escribirlo es que sea de alguna utilidad para futuros estudios interdisciplinarios de la relacion entre lo "micro" (es decir, los factores relacionados con la estructura productiva) y lo "macro" (es decir, la consistencia agregada). Este anhelo tiene sus rafces en la firme creencia de que buena parte de los secretos del crecimiento se encuentran escondidos en la compleja trama de las relaciones "micro-macro".

Si las formas más importantes de interacción 
"macro-micro" pudieran ser identificadas, sería posible analizar la manera en que diferentes escenarios macroeconómicos, caracterizados por diversas configuraciones de las variables agregadas, afectan positiva o negativamente la conducta de los agentes, la morfología institucional y, por ende, la eficiencia y el sendero de crecimiento de la estructura económica. Y sería también posible evaluar si cabe esperar que las reformas estructurales orientadas a cambiar las característi- cas del contexto en el cual se toman las decisiones microeconómicas actúen en favor, o bien en contra, de la consolidación de la estabilidad. Obviamente, dados nuestros objetivos, el análisis estará orientado primordialmente a dilucidar cuáles son las particularidades de esos mecanismos en el actual contexto latinoamericano, caracterizado por una estabilidad no consolidada y por una aceleración de las reformas estructurales orientadas a profundizar el rol de los mercados.

\section{Algunas precisiones sobre el problema que se analizará}

Es conveniente realizar algunas aclaraciones que nos ayudarán tanto a evitar confusiones como a delimitar nuestro objeto de análisis. Comencemos con una aclaración semántica. Utilizamos los términos "micro" y "macro" por razones de conveniencia y brevedad y sin mucho respeto por la tradicional división del trabajo en nuestra disciplina. En este estudio, el "problema macro" abarca las cuestiones de corto plazo que trata tradicionalmente la macroeconomía; pero también abarca otras que se relacionan con la consistencia entre el ahorro y la inversión en el largo plazo y que suelen analizarse en la teoría del crecimiento. Nuestro uso del término "micro", en tanto, hace referencia a una gama de características de la estructura económica que en un sentido es más amplia y en otro más estrecha que las que usualmente constituyen el objeto de estudio de la microeconomía. Es más amplia porque dentro del "problema micro" incluimos cuestiones normalmente tratadas en los enfoques institucionales y en la literatura sobre innovación y organización industrial. Es más estrecha porque, por ejemplo, no haremos referencia a cuestiones relacionadas con la teoría del consumidor.

Usualmente, la relación micro-macro se analiza en la literatura sobre microfundamentos de la macroeconomía. El propósito de esa literatura es estudiar cómo a partir de las conductas individuales determinadas por una estructura micro dada (recursos, tecnología, gustos y estructura de mercados), pueden fundamentarse las proposiciones más importantes de la macroeconomía de forma tal de contar con una teoría unificada. En definitiva, se trata de diluir el problema macroeconómico convirtiéndolo en un epifenómeno de las conductas micro, explicable por ende en el marco de una teoría general basada sólo en principios de conducta referidos a cada agente individual. A pesar de la cantidad de esfuerzo invertido, no se ha llegado a resultados que cuenten con un mínimo de consenso entre los especialistas. La discusión que sigue se relaciona sólo tangencialmente con esa problemática $\mathrm{y}$, digámoslo de una vez, no tenemos ninguna voluntad de reinventar la rueda.

A diferencia del enfoque de microfundamentos que trata de resolver un problema teórico, el que se usa aquí se origina en nuestra experiencia de estudios de casos en América Latina. Dada la falta de una estructura teórica integrada, en el análisis económico aplicado a casos concretos, los problemas de la estructura microeconómica y los macroeconómicos se estudian por separado. De manera consistente con lo anterior, en este estudio asumimos como premisa metodológica que los problemas macroeconómicos tienen entidad per se y que lo mismo ocurre con los problemas micro relacionados con la estructura productiva. A partir de ello -y basándonos en los hechos estilizados que hemos identificado a partir del estudio de casos- nos preguntamos cómo es la interacción entre ambos problemas: cómo determinadas estructuras micro afectan la estabilidad macroeconomica y cómo diferentes desequilibrios macroeconómi$\cos$ contribuyen a modelar una estructura micro determinada. Este enfoque no supone que la estructura micro está dada y que es invariable ante la ocurrencia de fenómenos de desequilibrio macroeconómico. 
Un ejemplo puede ser ilustrativo. En la literatura tradicional se supone que la inflación se produce por una falla de coordinación que hace que la demanda agregada supere a la oferta. Supongamos que ello se debe a un exceso de oferta de dinero. Para que el desequilibrio desaparezca sería suficiente que la autoridad monetaria realizara una política monetaria contractiva que resultara en una cantidad real de dinero exactamente igual a la demanda que surgirfa de la agregación de las demandas de cada uno de los agentes individuales. La estructura microeconómica es invariable ante el fenómeno de desequilibrio macroeconómico. Hay una y sólo una cantidad de dinero demandada compatible con cada nivel de inflación. Esto no siempre es consistente con los hechos estilizados de América Latina.

Supongamos que hay una tasa dada de inflación y la misma se acelera debido a un aumento de la tasa de emisión monetaria y que, como consecuencia, la demanda de dinero baja. Supongamos además que, mediante políticas de estabilizacion, las autoridades logran retrotraer la tasa de inflación y emisión al nivel previo. En un contexto como éste, en América Latina hemos observado muchas veces que la demanda de dinero no vuelve a su nivel original y, lo que es muy importante, que hay mercados financieros que simplemente desaparecen (típicamente los de más largo plazo). Esto es, para una misma tasa de inflación podría haber - aunque no necesariamente- dos niveles distintos de demanda de dinero y dos estructuras diferentes de mercados financieros. Si esto ocurre, ello significa que una vez que el desequilibrio macroeconómico es eliminado no se vuelve a la "misma" economía. El desequilibrio macro produce una mutación en la estructura micro. Este tipo de fenómenos no es privativo del campo monetario ya que tiene lugar también en el lado real. Así ocurre, por ejemplo, cuando las variaciones de la tasa de inflación producen variaciones permanentes en la duración media de los contratos o cuando desaparecen lisa y llanamente algunos tipos de contrato. En ambos casos, la estructura productiva puede sufrir mutaciones porque hay actividades productivas que devienen inviables si determinadas formas de contratación no están disponibles. ${ }^{1}$

El argumento no es que todo desequilibrio macro induce siempre mutaciones micro, sino que es posible identificar configuraciones de desequilibrio macro que efectivamente producen mutaciones estructurales. Es obvio que cualquiera sea el tipo de desequilibrio, lo

\footnotetext{
1 Sobre la relación entre inflación y contratos, véase Frenkel, 1990.
}

macroeconómico es per se importante porque tiene entidad causal suficiente como para condicionar las decisiones individuales de los agentes; independientemente de que el marco microeconómico se considere invariable o no. Pero cuando esas decisiones llevan a cambios en la tecnología o en la propensión a innovar, a destrucción de riqueza, a variaciones en la relación capi$\mathrm{tal} /$ producto o a transformaciones en las instituciones (cambios en el tipo de contratos, desaparición de mercados, etc), no puede asumirse que el marco micro dentro del cual se toman las decisiones individuales ha permanecido invariable.

La importancia de esta cuestión resulta clara cuando se observa la evolución de largo plazo de la estructura micro. Hay rasgos de la estructura económica que no pueden explicarse sólo en términos micro y sin hacer referencia al contexto macro en que tal evolución se produjo. Es necesario explicar, entonces, cuáles son las características que asumen algunos tipos especificos de desequilibrio macro que hacen que, como resultado de su ocurrencia, se produzcan mutaciones permanentes en la estructura micro. En este sentido, el objetivo no es sustituir el enfoque macroeconómico tradicional de corto plazo que supone un marco micro dado, sino complementarlo incluyendo en el análisis los efectos de largo plazo que los fenómenos de inconsistencia agregada pueden inducir en el marco micro.

En este artículo argumentaremos que para realizar tal extensión del análisis macro tradicional es necesario incluir, junto a los fenómenos de desequilibrio estable, aquéllos en los cuales pueden darse fenómenos de inestabilidad del desequilibrio. Esto es, hay que incluir escenarios macroeconómicos caracterizados no sólo por situaciones inestables explosivas (como hiperinflación) sino también, y fundamentalmente, aquéllos que muestran períodos prolongados de desequilibrio que no devienen explosivos (por ejemplo, lapsos extendidos de inflación de tres dígitos) o que muestran una sistemática propensión a generar desequilibrios pronunciados y recurrentes de forma tal que ello se convierte en una característica inherente del contexto macroeconómico. Desde nuestro punto de vista, es justamente la percepcion por parte de los agentes económicos de que viven en una economía con rasgos de inestabilidad macroeconómica la que los induce a poner en práctica cambios de conducta que terminan por provocar mutaciones estructurales en el entomo micro.

De hecho, la importancia de esta cuestión está ya implícita en el lenguaje mismo con que se discuten los 
fenómenos macroeconómicos en América Latina. Cuando en la literatura se hace referencia a problemas macroeconómicos en los pafses desarrollados, se habla de desequilibrio macroeconómico. Cuando se discute de macroeconomía en Latinoamérica, sin embargo, se cambia sin mucha discusión la palabra equilibrio por estabilidad y se discurre sobre el problema de la estabilidad macroeconómica y las políticas de estabilización. En el primer caso, implícitamente se asume que la economía es estable y el problema de política macroeconómica es cómo hacer más rápida la vuelta al equilibrio de una economía inherentemente estable. En el segundo caso, no se asume -creemos que correctamente- que la economía sea estable. El problema no es acelerar la vuelta a un equilibrio predefinido sino cómo encontrar políticas para convertir en estable a una economía inestable. Se trata de definir una nueva configuración del equilibrio y nuevas formas de ajuste dinámico en desequilibrio.

Es justamente por esta razón que la política de estabilización suele incluir elementos de reforma estructural y que es tan difícil separar ambos componentes. Asimismo, este es un motivo importante por el cual es necesario adoptar un enfoque macroeconomico que incluya cuestiones normalmente tratadas en la teoría del crecimiento. Si la economía tiene rasgos de inestabilidad es necesario cambiar los parámetros que la definen para hacerla estable. Es decir, reformar parte de su estructura. Y si se obtiene éxito en la estabilización, la economía estabilizada no será estrictamente la misma economía generadora del desequilibrio macroeconómico que llevó a aplicar la política de estabilización. Las economías inherentemente estables no se estabilizan, se equilibran. Son las inestables las que deben estabilizarse. Esto es, las que deben ser transformadas estructuralmente para que muestren senderos estables de retomo al equilibrio y por ende sean posibles de ser equilibradas.

La estrecha relación entre estabilización y reforma estructural que plantean los fenómenos de inestabilidad genera a su vez problemas adicionales. Al diseñar las políticas de cambio estructural que deben acompanar a la estabilización, ¿cuáles son los parámetros estructurales que deben ser cambiados, qué características de la estructura micro son las que generan la propensión a la inestabilidad y cómo afectan esos cambios a la capacidad de crecimiento de la economía? Esto obliga a analizar los efectos que lo micro tiene sobre lo macro.

En suma, en el enfoque que adoptamos en este estudio, la relación entre lo micro y lo macro es concebida como un camino de doble vía, de modo que tenemos por delante dos tareas. La primera es analizar por qué existe un "problema" macroeconómico con una especificidad y una entidad que le son propias, y que amerita un tratamiento (analíticamente) separado del resto de las cuestiones atinentes a la evolución de las actividades productivas. Particularmente, es crucial tener una idea de por qué el desequilibrio macroeconómico y, frecuentemente, la aparición de fenómenos de inestabilidad, han afectado tan sensiblemente el ritmo de crecimiento y la morfología de la estructura productiva de América Latina. La segunda tarea es examinar de qué manera los desbalances existentes en la estructura productiva así como un mayor desarrollo y sofisticación de la misma, podrían contribuir a la desactivación de los factores estructurales que están en la base de la propensión de las economías de la región a generar fuertes desequilibrios macroeconómicos en forma recurrente, y que suelen asumir el carácter de inestables.

\section{III}

\section{De lo macro a lo micro: la especificidad del problema macroeconómico y}

\section{las mutaciones estructurales}

Lo que dio lugar a la aparición de la literatura sobre los microfundamentos es que, partiendo de los principios microeconómicos, no es posible deducir como teorema los hechos estilizados que se observan a nj- vel macroeconómico, como el desempleo o la importancia de las variables nominales en la determinación del nivel de actividad agregado. La imposibilidad de resolver esta cuestion hizo que, como hipótesis de 
trabajo, el problema macroeconómico se concibiera con entidad propia e independiente de las conductas individuales. Para definir tal entidad, el análisis macroeconómico identifica un grupo de variables fundamentales que determinan la evolución de la economía a nivel agregado. Tales variables son las asociadas con la evolución del sector externo, de las cuentas fiscales y del balance entre el ahorro y la inversión globales. ${ }^{2}$ Cuando se producen cambios pronunciados y no anticipados en estas variables fundamentales aparecen fallas de coordinación entre los planes individuales y desequilibrios de entidad macroeconómica.

El núcleo central del problema macroeconómico es la existencia de esas fallas de coordinación. Cuando éstas se producen, ello determina que no todos los agentes económicos estén en condiciones de transar ex post en los mercados las cantidades que habían planeado ex ante. Existe desequilibrio macroeconómico porque existen fallas de coordinacion a nivel agregado.

La principal distorsión que plantea una situación de desequilibrio es que los agentes se ven obligados a realizar transacciones que pueden superar o ser menores en magnitud a las deseadas, y esto afecta la restricción de presupuesto y la posición de riqueza de tales agentes. ${ }^{3}$ Como consecuencia, éstos se ven compelidos a llevar a cabo ajustes no previstos con el objeto de recomponer el equilibrio deseado en su hoja de balance, $y$ tales ajustes pueden abarcar tanto el lado real (reasignación de factores) como el financiero (decisiones de cartera), y tanto los flujos (decisio-

2 En la discusión sobre estabilización y crecimiento en América Latina, el rol crucial de estos tres factores llevó al desarrollo de la literatura basada en los modelos de "tres brechas": la fiscal, la externa y la interna (de ahorro/inversión), Véase, por ejemplo, Fanelli, Frenkel y Taylor, 1992.

3 En la literatura de desequilibrio tradicional (Leijonhufvud, 1968; Benassy, 1982) se ponen de relieve las transacciones que no se realizan debido a fallas de coordinación. Sin embargo, en otro lugar hemos argumentado que este énfasis es injustificado (Fanelli, 1988). Sobre todo en el plano financiero, donde en situaciones de desequilibrio típicamente los agentes sobreendeudados tienen la posibilidad de atrasarse en los pagos, obligando consecuentemente a los acreedores a prestar más que lo que ex ante estaban dispuestos a prestar. Este tipo de situación fue muy comán durante la crisis de la deuda en que varios países latinoamericanos entraron en moratoria de pagos. Cuando se hace hincapié en las transaciones que no se realizan, el esquema de racionamiento en desequilibrio que se utiliza es el de que "Inanda el brazo corto del mercado" (por ejemplo, si existe exceso de oferta se transan las cantidades determinadas por la demanda). Cuando se permite que se hagan más transacciones que las deseadas, el esquema de racionamiento puede basarse en que "manda el brazo largo del mercado" o una combinación lineal de las cantidades determinadas por el brazo corto y el largo. En el primer caso todas las transacciones son voluntarias, en el segundo pueden existir transacciones forzosas o no voluntarias. nes dentro del período) como los stocks (reasignaciones intertemporales de recursos). Estos problemas aparecen ante cualquier situación de desequilibrio. Pero cuando ellos se deben a fallas de coordinación a nivel agregado, el dato clave es que habrá un número macroeconómicamente importante de agentes realizando transacciones en desequilibrio y ajustando, por ende, sus hojas de balance.

A su vez, las consecuencias de esos ajustes sobre la hoja de balance de otros agentes -que en un principio tal vez no hayan sido afectados- no se pueden ignorar, ya que serán de magnitud no despreciable. En otras palabras, se producirán efectos de derrame sobre otros mercados que tenderán a profundizar los impactos del desequilibrio en un mercado o mercados determinados. Además, como las transacciones en desequilibrio pueden afectar no sólo a los flujos sino también a la posición de acervos (stocks), el efecto de derrame puede tener consecuencias intertemporales importantes. El desequilibrio puede "leerse" en la hoja de balance de cada agente si se observan las decisiones sobre acervos y flujos que deberá tomar para solucionar su desequilibrio, y es justamente por ello que las fallas de coordinación son fundamentales para entender por qué lo macro afecta a lo micro.

Los canales de transmisión a través de los cuales las fallas de coordinación a nivel agregado terminan afectando las decisiones de los agentes son variados y numerosos. En función del tipo de análisis que aquí nos preocupa, sin embargo, basta con una categorización general de los canales fundamentales. El mecanismo de transmisión básico en una economía de mercado, obviamente, es el que opera a través del sistema de precios. Esto es, a través de variaciones de los precios relativos tanto en el lado real como en el financiero. Sin embargo, el sistema de precios no actúa en forma óptima porque los precios no son completamente flexibles; la información no es perfecta; hay factores que debilitan la competencia, y hay rigideces y fricciones en la reasignación de recursos. En realidad, es por estas imperfecciones de mercado que se producen fallas de coordinación y desequilibrios que llevan a la generación de sefiales falsas de precios relativos. El hecho de que el mecanismo de precios no sea óptimo en tales circunstancias, lleva a sobrecorrecciones o subcorrecciones de precios que dan lugar a que se produzcan variaciones de cantidades (ofertadas o demandadas) y decisiones financieras erroneas. Es decir, decisiones que impedirán que los agentes puedan transar las cantidades planeadas en los mercados reales y financieros. Los fenómenos 
de desempleo en el lado real y las situaciones de sobreendeudamiento o severa restricción de liquidez en el lado fínanciero son los indicadores más visibles de este tipo de situaciones.

Cuando los agentes aprenden que sus decisiones pueden ser erróneas porque los precios no incluyen toda la información relevante o incluyen información que puede ser errónea, buscan información complementaria a la brindada por los precios relativos. Como los efectos más visibles del desequilibrio operan sobre las cantidades reales transadas y sobre las variaciones en la posición financiera de los agentes, aparecen dos conjuntos nuevos de señales que es preciso tomar en cuenta en las decisiones individuales: la información de cantidades reales transadas en los mercados y la información de cantidades brindada por los indicadores financieros elaborados sobre la base de la hoja de balance de los agentes. En función de esto, es necesario incorporar dos tipos de mecanismos de transmisión adicionales al de precios que afectan las decisiones microeconómicas y a través de los cuales, por ende, lo macro afecta a lo micro. Ellos son, por una parte, los que operan a través de los indicadores de cantidades en el lado real (por ejemplo, desempleo y capacidad ociosa) y, por otra, los que actúan a través de variaciones en los indicadores financieros de cantidad. Entre estos últimos los hay de flujo/flujo (como el indicador pago de intereses/ventas); de acervo/acervo de corto plazo (por ejemplo, el indicador de liquidez activos líquidos/ deuda de corto plazo exigible) y de largo plazo (por ejemplo endeudamiento/patrimonio neto), y de acervo/ flujo (por ejemplo, ventas/deuda).

Una vez definida la especificidad del problema macroeconómico sobre la base de las variables fundamentales y las fallas de coordinacion por una parte, y de los mecanismos de transmision entre el desequilibrio agregado y las conductas microeconómicas por otra, queda por analizar cómo es que los efectos del desequilibrio económico inducen, bajo ciertas circunstancias, mutaciones en la morfología y en la evolución en el tiempo de la estructura micro. Ya dijimos que en esto los fenómenos de inestabilidad son fundamentales. Ahora queremos mostrar cómo ello se correlaciona con la magnitud cuantitativa de las fallas de coordinación, su duración temporal y su recurrencia.

La coordinación completa (es decir, la ausencia de problemas macro) sólo se produce en un contexto de equilibrio general y tal contexto es una construcción ideal. No obstante, es una construcción útil pues la referencia a esa situación permite considerar diferentes grados de desequilibrio macroeconómico. Aun- que las economías se encuentran siempre en situación de desequilibrio, un grado reducido de desequilibrio puede considerarse poco relevante en la determinación de su funcionamiento y por ende la estilización de equilibrio puede ser útil para modelarlo. El problema macroeconómico aparece en plenitud cuando la magnitud del desequilibrio en las variables fundamentales es tal que afecta en forma esencial el funcionamiento de la economía.

En el análisis macroeconómico tradicional, el desequilibrio agregado es concebido como un fenomeno temporal y se hace hincapié en la búsqueda de herramientas de intervención optimas - sobre todo referidas al uso de las políticas fiscales, monetarias y cambiarias - capaces de solucionar las fallas de coordinación, minimizando a la vez el período durante el cual la economía funcionará en desequilibrio. Más allá de las recientes discusiones sobre si tales políticas son o no necesarias, el análisis tradicional supone que el desequilibrio es temporal. Sea porque la economía se autorregula adecuadamente sin necesidad de intervenciones de política, sea porque no lo hace pero es posible acelerar el recorrido a través del sendero que lleva al equilibrio mediante un proceso de sintonizacion fina. Si este fuera el caso, las consecuencias a nivel micro de los desajustes macro no serían excesivamente relevantes.

Este enfoque, sin embargo, resulta demasiado estrecho cuando se analiza el problema macroeconómico en economías como la latinoamericana típica. La razón básica es que los problemas macroeconómicos que enfrenta la región suelen revestir características diferentes de los que típicamente se observan en las economías desarrolladas. Las diferencias más marcadas se asocian con la magnitud, la duración temporal y la recurrencia de los desequilibrios macroeconómicos. Estos rasgos se manifiestan de manera múltiple y variada en cada economía en particular. Sin embargo, hay un rasgo que sintetiza la diversidad de factores que contribuyen a determinar la propensión de una economía a generar fallas de coordinación: el grado de volatilidad e impredictibilidad estocástica en la evolución de las variables fundamentales. Cuanto más volátil y más difícil de modelar es el proceso estocástico que genera la evolución observada de las variables fundamentales, mayor será la dificultad para anticipar tal evolución, mayor la incertidumbre y, por ende, mayor la propensión de la economía a generar desequilibrios externos, fiscales o entre el ahorro y la inversión. En un contexto tal, habrá períodos recurrentes durante los cuales se producirán fallas de coordinación importantes. 
En una economía que muestra estas características en la evolucion de sus variables fundamentales, habrá a nivel microeconómico conductas que estarán ausentes cuando ello no ocurra. Lo que queremos resaltar es que una cosa es pasar por una etapa de desequilibrio y otra vivir en una economía propensa a generarlo en forma sistemática. En este último caso, a diferencia de lo que se subraya en el análisis macroeconómico tradicional, como el desequilibrio macroeconómico se percibe como un fenómeno recurrente, aparecen como rentables conductas de adaptación microeconómica que no lo serían si el desequilibrio fuera un fenómeno esporádico y pasajero. Es razonable pensar que, en una economía estructuralmente propensa a generar desequilibrios macroeconómicos, la influencia de lo macro sobre la estructura micro será mucho más permanente y visible. Ya hemos visto que los mecanismos de transmisión macro-micro operan a través del sistema de precios, la información de cantidades y la posición financiera de los agentes. Vale la pena, entonces, detenerse brevemente en las características particulares que tales canales muestran en una economía con propensión al desequilibrio recurrente e inestable a nivel macroeconómico.

Empecemos con la operatoria a través del sistema de precios. Como en las economías de mercado le corresponde a los precios relativos la labor primordial de eliminar los desequilibrios, una consecuencia importante de la volatilidad de las variables fundamentales será una más alta y pronunciada variabilidad de los precios relativos. Esto es, en los precios relativos que el análisis económico identifica como claves justamente porque están asociados a las variables fundamentales (tipo de cambio real, salarios reales, tasas de interés, etc.). Si las señales de precio que reciben los agentes son más volátiles, el grado de incertidumbre que rodeará el proceso de toma de decisiones tenderá a ser elevado $\mathrm{y}$, en consecuencia, será mayor la probabilidad de que el individuo tome decisiones erróneas que lo lleven ex post a una situación de desequilibrio.

La evolución de las cantidades transadas en desequilibrio no es independiente de lo anterior. Ya vimos que debido a imperfecciones (rigideces, etc.) no todo el ajuste se produce vía precios. En consecuencia, las cantidades mostrarán también una evolución más volátil en un contexto en que es difícil anticipar el comportamiento de los precios relativos. Habrá cambios bruscos y no anticipados de cantidades durante el proceso de ajuste, dando lugar de esa forma a ciclos rápidos y recurrentes de recesión/expansión que agregarán incertidumbre al marco de decisión microeconómico.

Por último, como en una economía monetaria a toda transacción del lado real le corresponde otra del lado financiero, los indicadores de la hoja de balance financiero también tenderán a mostrar cambios marcados y no anticipados. En realidad, las variaciones de precios y cantidades no anticipadas pueden poner en desequilibrio a los agentes aún cuando éstos "no decidan nada" en el plano financiero. Ello ocurre, por ejemplo, cuando las devaluaciones no anticipadas generan transferencias de riqueza también no anticipadas que suelen cambiar fuertemente la posición financiera de los agentes (por ejemplo, convirtiendo una posición financiera sólida en una altamente especulativa o Ponzi) o cuando profundas recesiones llevan a posiciones financieras insostenibles.

Es justamente esta forma diferenciada en que operan los mecanismos de transmisión micro-macro lo que explica que, en una economía con tendencia a generar inestabilidad, los desequilibrios macro produzcan mutaciones micro. En realidad, las mutaciones que se observan están de una u otra manera relacionadas con el siguiente hecho: en una economía de alta incertidumbre macroeconómica donde es más fácil equivocarse, la flexibilidad para cambiar decisiones del pasado tiene un premio económico. ${ }^{4}$ Empíricamente, la forma más directa de verificar este hecho es observar la enorme diferencia de riesgo entre el nivel de las tasas de rendimiento de corto y largo plazo. Suelen observarse situaciones en las que tal diferencia se torna infinita para algunos plazos haciendo desaparecer de tal forma los mercados de crédito de largo plazo.

Esta suerte de preferencia extrema por la flexibilidad tiene consecuencias fundamentales sobre la asignación de recursos reales, la morfología financiera y - a través de sus efectos en la inversión y la capacidad de innovación- sobre el crecimiento. Más adelante utilizaremos este resultado, que es una de las conclusiones centrales de nuestro análisis porque afecta de manera directa el desarrollo productivo. Por ahora nos interesa destacar dos puntos adicionales que tienen que ver con la dinámica de ajuste en desequilibrio y las restricciones que una economía inestable pone a la política macroeconómica.

En una economía inestable, las reacciones de los individuos no sólo producen mutaciones en la estructura micro; tales mutaciones pueden generar además

\footnotetext{
4 Nuestro uso del concepto de flexibilidad está inspirado en Hicks 1974.
} 
mecanismos de ajuste en desequilibrio que lleven a senderos dinámicos explosivos. Por ejemplo, se produce un déficit fiscal y la inflación se acelera. Para cubrirse de los efectos de esa aceleración los individuos reaccionan, acortando en forma generalizada la duración de los contratos. Este hecho achica el horizonte intertemporal de decisión microeconómica. Hay proyectos de inversión que no se realizan y activos financieros que dejan de demandarse. Como consecuencia, cae el nivel de actividad y es más difícil para el gobierno y el sector privado colocar deuda, incluso a plazos muy cortos. Un nivel de actividad más bajo reduce la recaudación tributaria, el déficit fiscal aumenta y en el contexto de una caída en la demanda de bonos públicos, la única forma de financiar al gobierno es aumentar la tasa de emisión monetaria. Esto acelera la inflación, y así sucesivamente. El sendero de ajuste en desequilibrio comienza a mostrar signos de explosividad y esto convalida como correcta la decisión individual de acortar los contratos para adaptarse a una economía inestable. El ajuste en desequilibrio lleva a consolidar la mutación a nivel micro.

Estos mecanismos de realimentación del desequilibrio y consolidación de las mutaciones micro también afectan la efectividad de las políticas económicas. Cuando se producen cambios frecuentes y no anticipados en el sendero temporal de las variables fundamentales, las autoridades se ven obligadas a efectuar cambios frecuentes y radicales en los instrumentos de política, tratando de compensar los efectos desequilibrantes. En este contexto, es muy improbable que las autoridades logren un prestigio suficiente para dar credibilidad a sus políticas.

Esto tiene dos consecuencias negativas. En primer lugar, es difícil que los agentes económicos muestren rapidez y flexibilidad en adaptar sus conductas cuando el gobiemo anuncia cambios radicales de política; en un contexto incierto, las conductas conservadoras y defensivas pueden ser las más rentables, en cuanto garanticen mayor flexibilidad. En segundo lugar, se obliga al gobierno a exagerar en la elección de los objetivos cuantitativos buscados con sus políticas: los anuncios deben ser dramáticos si han de cambiar la conducta de agentes conservadores.

Ambos hechos son una fuente permanente de fallas de coordinación macroeconómica e inestabilidad en los países de la región. Si la conducta de los agentes no cambia en el sentido que desean las autoridades y, además, las políticas buscan objetivos dramáticos, las consecuencias en términos de desequilibrio macroeconómico y desarticulación productiva pueden ser altamente distorsivas. Cuestiones de este tipo han sido típicas, por ejemplo, de los ciclos de política economica de proteccion-apertura-crisis de balance de pagos-protección que se dieron en Argentina, Chile y otros países. Para ser creíbles, las autoridades lanzan un programa rápido y muy ambicioso de apertura comercial. Con ello esperan que el sector privado tome la apertura como un hecho permanente e invierta en la reconversión. Como la apertura genera una explosión de las importaciones y un déficit insostenible en la cuenta corriente, el sector privado anticipa que el régimen no podrá ser mantenido y no invierte en reconvertirse. En tal contexto, las exportaciones se estancan y las importaciones suben. Cuando se toma imposible financiar el déficit de cuenta corriente resultante, con el objeto de garantizar un mínimo de estabilidad macroeconómica las autoridades se ven obligadas a volver a imponer no sólo altos aranceles, sino directamente prohibiciones y cuotas de importación. Ex post, las conductas defensivas son convalidadas como correctas por la política economica.

\section{IV}

\section{De lo micro a lo macro: desbalance estructural y estabilidad macroeconómica}

No todos los países muestran el mismo grado de inestabilidad macroeconómica, porque en cada país la volatilidad de las variables fundamentales y los mecanismos específicos de ajuste ante el desequilibrio difieren. Tales particularidades se relacionan estrechamente con las características estructurales de cada economía. Es por esto justamente que los factores micro influyen en la determinación de la capacidad de la economía para procesar shocks macroeconómicos con mayor o menor eficiencia. 
La estructura económica está compuesta por una base productiva (tecnología, recursos, capacidad de innovación), individuos, mercados e instituciones. Por lo tanto, la capacidad de la economía para absorber shocks que se traducen en fallas de coordinación puede evaluarse en función de: i) la cantidad y calidad de los mecanismos de autorregulación existentes (mercados e instituciones) para amortiguar y distribuir los shocks en el tiempo; y ii) las características del sistema productivo, que lo hacen más o menos flexible en la reasignación de los factores de producción con el objeto de adaptarse a situaciones nuevas (por ejemplo, posteriores al shock).

En función de lo anterior, las economías podrían ordenarse de acuerdo al grado de desarrollo de los mercados y las instituciones, y la diversificación de su estructura productiva. Si se toman, por ejemplo, las economías con algún grado significativo de desarrollo industrial, los dos criterios mencionados podrían utilizarse para crear un orden en el cual las economías capitalistas desarrolladas estarían en un extremo y las ex economías socialistas en transformación en el otro, en tanto que las economías latinoamericanas de mayor tamaño ocuparían lugares entre ambos extremos.

El criterio i) hace depender la capacidad de autorregulación del grado de desarrollo de los mercados y las instituciones. Tanto la estructura de los mercados como las instituciones directa o indirectamente relacionadas con la actividad económica son cruciales, porque de ellas depende la capacidad de administrar la incertidumbre inherente a todas las actividades económicas, y porque pueden contribuir significativamente a suavizar los efectos desequilibrantes generados por la volatilidad.

Cuanto mayor sea el grado de desarrollo de la estructura de mercados, más aceitado resultará el proceso de determinación de precios y, por lo tanto, menor será la probabilidad de que ocurran desequilibrios duraderos y procesos inestables. Un sistema de mercados "completo" permitiría que la eliminación de los desequilibrios spot se distribuyese en el tiempo a través de la operación de los mercados futuros, y que la incertidumbre se procesase a través de la operatoria de los mercados de seguros. En este sentido, el modelo ideal de referencia es la estructura de mercados y de información "perfecta", que cuenta con todos los mecanismos necesarios para garantizar la coordinación completa de los planes y decisiones descentralizadamente decididos por los individuos y en la cual la reasignación de los recursos ante "noti- cias" que cambian el contexto de la información se hace en forma más o menos instantánea y sin incurrir en costos significativos.

Ninguna economía real tiene tales características, pero las economías capitalistas desarrolladas poseen las estructuras que más se aproximan a esa estilizacion teorica. Aunque la estructura de esas economías exhibe numerosas fallas de mercado, es usual que existan sustitutos próximos de los mercados que faltan. Por ejemplo, la ausencia de mercados de futuro generalizados tendrá menos efectos cuanto más desarrollados estén los mercados de capital de diferentes plazos, y si bien no existen mercados para asegurarse ante todo tipo de tiesgo, los mercados de seguro para diferentes clases de riesgo están mucho más desarrollados que en la economía latinoamericana típica.

De lo anterior se concluye que el carácter más o menos "completo" de la estructura microeconómica de mercados es relevante para la dinámica macro, porque existe una correlación estrecha entre los problemas de fallas de coordinación macroeconómica y la magnitud de las fallas de mercado. A mayor número e importancia de las fallas de mercado, mayor debilidad $\longrightarrow$ ausencia - de mecanismos de mercado para la amortiguación del desequilibrio, y mayor en consecuencia la tendencia de las economías a generar desequilibrios y senderos de ajuste dinámico que tiendan a reproducir o amplificar los desbalances en lugar de corregirlos.

El grado de desarrollo de las instituciones relacionadas con la actividad económica afecta la probabilidad de que ocurran fallas de coordinación macroeconómica, por varias razones. Vale la pena mencionar dos. La primera es que existen arreglos institucionales que pueden suplir fallas de mercado como la inexistencia de algunos mercados de futuro $o$ de seguros claves y en tal carácter, son mecanismos relevantes de reducción de la incertidumbre. Estas instituciones tienen importancia en el establecimiento de relaciones de clientela para reducir la incertidumbre en las relaciones entre firmas que operan en un mundo de mercados spot; para suplir las fallas en el mercado de trabajo o las externalidades generadas en el proceso de aprendizaje financiado por firmas particulares; para proveer sustitutos de mercados de crédito inexistentes, a través de bancos estatales o a través de las relaciones crediticias cliente/proveedor/firma, etc.

La segunda razón es que la fortaleza institucional reduce la volatilidad de las variables fundamentales. Cuanto más desarrolladas sean las instituciones 
del sector público (por ejemplo, la estructura impositiva), menor sera la probabilidad de que ocurran shocks fiscales que lleven al desequilibrio macroeconómico. Cuanto más eficiente la supervisión bancaria y los mecanismos de selección para la asignación de crédito por los bancos comerciales, menor la probabilidad de que se produzca una crisis financiera, con las consecuentes externalidades negativas para el equilibrio macroeconómico. Además, el desarrollo institucional es relevante porque existe una correlación entre éste y el grado de estabilidad del marco jurídico y de regulaciones, lo cual es a su vez determinante en la evolución de la inversión en particular y de la incertidumbre en general. En una economía basada en la propiedad privada, la seguridad jurídica no solo es una condición necesaria de la existencia misma de los mercados, sino también de la inversión que garantice la reproducción del sistema económico.

El criterio ii) hace directa referencia a la estructura productiva. En general, cuanto menor la diversificación y sofisticación productiva, menor la capacidad para reasignar rápidamente los recursos hacia nuevas actividades al producirse shocks permanentes. En las economías poco desarrolladas en lo productivo, existe una mayor cantidad de rigideces que impiden una rápida reconversión de la estructura productiva. Esto hace que el proceso de transición y de ajuste tras el shock sea de mayor duración y, en consecuencia, será mayor el período durante el cual la economía funcionará en desequilibrio. Es importante destacar que - por lo menos empíricamente--. está probado que los factores i) y ii) no son independientes y que este hecho tiende a reforzar los mecanismos de reproducción y prolongación del desequilibrio.

En efecto, se observa en primer lugar que la falta de desarrollo productivo se correlaciona positivamente con la falta de desarrollo de los mercados financieros y de capital. Así, la propensión a la inestabilidad se refuerza porque la rigidez de la estructura productiva determina que los procesos de transición sean largos, y la falta de mercados de capital hace que sea muy difícil financiar procesos largos de reconversión productiva. El resultado es que en la economía latinoamericana típica, por ejemplo, haya prolongados períodos de retracción productiva que no necesariamente llevan a una reconversión exitosa del aparato productivo, sino a perdidas netas de capacidades de producción que habían sido adquiridas. Ejemplo paradigmático en este sentido son los procesos de apertura económica de shock caracterizados por la desaparición de partes importantes del sector industrial, sin que aparezcan nuevas especializaciones y economías de escala en determinados segmentos del sistema industrial, debido a la falta de financiamiento para la reconversión.

En segundo lugar, también se observa empíricamente que existe una correlación entre el grado de desarrollo productivo y la sofisticación de las instituciones asociadas con la actividad económica. Esto hace que el desarrollo de instituciones orientadas a sustituir a los mercados faltantes - debido a fallas de mercado- también se retrase; así la capacidad para procesar la incertidumbre se relaciona directamente con la escasa sofisticación de la estructura productiva. En realidad, a la luz de los escritos sobre organización industrial, este hecho no resulta sorprendente. De ellos surge que el desarrollo de las instituciones orientadas al manejo de la incertidumbre al margen del mercado, como las relaciones entre clientes, proveedores y firmas, tiende a ser mayor en los sectores industriales de mayor sofisticación, donde los precios desempeñan un rol mucho menor en la asignación de los recursos que, por ejemplo, en los mercados de productos básicos y materias primas en general. ${ }^{5}$ Así, a menor sofísticación productiva, menor será la presencia económica de estas instituciones y mayor el rol de la incertidumbre y la inestabilidad.

Dos ejemplos simples pueden servir para aclarar lo que hemos tratado en esta sección. El primero se refiere a los efectos multiplicadores e ilustra el papel de los cambios de la estructura micro en la determinación de la dinámica de ajuste macro.

La magnitud del efecto multiplicador del gasto es mayor en una economía como la argentina que en la economía estadounidense actual. Es conocido que el efecto multiplicador se ha ido debilitando en esta última, hasta el punto de que su misma existencia ha sido puesta en duda desde principios de los años sesenta. En cambio, ese efecto se observaba claramente en los años treinta. Esta transformación en la dinámica del comportamiento macroeconómico a lo largo del tiempo puede atribuirse a la mayor complejidad de la estructura económica (desarrollo de los mercados e instituciones y acumulación de riqueza). En pocas palabras, los asalariados poseen ahora mayor riqueza, cuentan con el seguro de des-

\footnotetext{
5 Scherer (1980) aporta una cantidad abrumadora de hechos estilizados atinentes a la organización industrial que apoyan esta afirmación.
} 
empleo y tienen mayor acceso al crédito, de modo que una reducción transitoria de su ingreso corriente afecta menos su gasto de consumo que sesenta años atrás. La economía argentina, en cambio, tiene al respecto una estructura más parecida a la de Estados Unidos en los años treinta. Una reducción del ingreso corriente de los asalariados argentinos (más pobres que sus homólogos estadounidenses y sin seguro de desempleo generalizado) les impone una restricción de liquidez que limita su gasto en consumo. Se observan entonces las repercusiones de los "efectos ingreso" que determinan la magnitud del multiplicador. Esos mismos mecanismos, dependientes de la estructura de la economía, determinan también otros comportamientos diferentes ante situaciones de desequilibrio: por ejemplo, los efectos que se observan ante una devaluación. La diversidad estructural - - en particular en lo relativo a la diversificación de la base productiva - determina que la devaluación sea recesiva en Argentina y expansiva en los Estados Unidos.
El segundo ejemplo se refiere a la falta de diversificación productiva como causa directa del grado de volatilidad macroeconomica. Uno de los determinantes principales de la variabilidad del saldo de la cuenta corriente en América Latina es la variabilidad de los términos del intercambio. Como las exportaciones muestran un bajo nivel de diversificación fruto de la falta de competitividad de los sectores más dinámicos de la industria (Guerrieri, 1993), la cuenta comercial suele sufrir cambios de significación ante la ocurrencia de shocks externos negativos. La falta de diversificación productiva hace que la volatilidad de los términos del intercambio se traduzca en forma directa en volatilidad de las variables fundamentales que afectan al sector externo. El depender de pocos productos de exportación impide suavizar la volatilidad mediante la diversificación del riesgo implícita en una estructura exportadora diversificada. De esta forma, la falta de desarrollo productivo se convierte en forma directa en una fuente de volatilidad macroeconómica.

\section{V}

\section{La interacción micro-macro, la estructura}

\section{productiva y el crecimiento}

Hasta aquí hemos analizado como la inestabilidad ma-
cro puede inducir mutaciones en la estructura micro y
cómo las debilidades micro -en la estructura pro-
ductiva, de mercados e instituciones- pueden gene-
rar fallas de coordinación. La división que hemos he-
cho en el tratamiento de estas dos cuestiones obedece
exclusivamente a razones metodologicas y de exposi-
ción. Para evitar confusiones, nos gustarfa recalcar
que, a nivel empírico, la regla general es que hay una
miríada de interacciones y mecanismos de retroali-
mentación entre lo macro y lo micro que hacen que,
de hecho, se produzca una suerte de determinación
simultánea de las características micro y macro de
una economía específica. En el análisis de casos con-
cretos es muy difícil determinar si una economía mues-
tra, digamos, graves fallas de mercado debido a su
inestabilidad macroeconómica o si, por el contrario,
es la falta de algunos mercados claves -como el de capitales de largo plazo- el que genera la situación de inestabilidad agregada. ${ }^{6}$

Esto no quiere decir, obviamente, que en una situación concreta el resultado evolutivo final del proceso de interacción de los factores micro y macro no pueda analizarse separando la incidencia de cada uno

\begin{abstract}
6 Normalmente ocurre que ambos fenómenos tienden a retroalimentarse. Por eso en períodos de crisis aguda - cuando tanto la inestabilidad como la falta de mercados clave pasan un umbral crítico- es tan difícil encontrar una salida. Las politicas de estabilización no acompañadas de otras de reforma estructural tienden a fracasar debido a las deficiencias existentes en la estructura de los mercados, y las de reforma estructural sin las de estabilización fallan debido a la incertidumbre de un contexto macroeconómico desequilibrado. Esto es un argumento adicional en favor de que las estrategias de estabilización vayan acompañadas de políticas de reforma y tambien en favor de que no se implementen las políticas de reforma que pueden llevar a agudizaciones del desbalance macroeconomico (Fanelli y Frenkel, 1994).
\end{abstract}


de esos dos factores. En realidad, la posibilidad de realizar esta separación analítica entre esos factores es fundamental para comprender las restricciones al crecimiento que una economía enfrenta en una coyuntura económica dada. Para completar nuestro enfoque metodológico queremos justamente detenernos en las implicaciones que este tiene para el crecimiento.

Para presentar la cuestión de las restricciones al crecimiento de manera sucinta, en otro trabajo (Fanelli y Frenkel, 1994) las hemos sistematizado en función de cuatro problemas que, de una u otra manera, las economías deben resolver para estar en condiciones de crecer. En primer lugar está el problema smithiano; para crecer de manera rápida y sostenible una economía debe generar una tasa de ahorro que le permita mantener una alta tasa de inversión financiada con sus propios recursos. El segundo es el problema keynesiano, que resalta que no sólo es necesario ahorrar, sino que también hay que contar con mecanismos eficientes para intermediar entre el ahorro y la inversion, esto es, contar con una estructura desarrollada de mercados e instituciones capaces de garantizar que el esfuerzo de ahorro se transforme efectivamente en inversión productiva. El tercero es el neoclásico, que hace hincapié en que los recursos disponibles deben ser asignados eficientemente para maximizar la tasa de crecimiento: no sólo importa cuánto se invierte sino también la eficiencia de la inversión. Por último, el problema schumpeteriano hace referencia a la creatividad como motor del crecimiento y pone de relieve el rol de los empresarios y las firmas que innovan (en el plano tecnológico, organizacional, etc.). ${ }^{7}$

Las interrelaciones micro-macro que hemos desarrollado son fundamentales para los últimos tres problemas del crecimiento mencionados, ${ }^{8}$ ya que contribuyen a modelar los mercados y las instituciones de intermediación financiera entre el ahorro y la inversion; determinan la eficiencia con que la información económica pertinente es procesada -a través de las señales de precios, cantidades y financieras- contribuyendo así a optimizar la asignación de los recursos, y afectan de manera marcada la capacidad de innovación de la economía, al influir sobre la conducta indjvidual de los agentes y sobre las instituciones que conforman el sistema nacional de innovación. ${ }^{9}$

7 Nelson (1991 y 1992) examina el rol del empresario frente al de la firma en el proceso de innovación y como agente de la, "destruc. ción creativa".

8 En este artículo no estamos interesados en tratar el problema de cómo afectan a la restricción de ahorro.

9 Sobre los sistemas nacionales de innovación, véanse por ejemplo Nelson (1993), Mowery (1993) y Johnson y Lundvall (1988).
Muchos de estos efectos pueden examinarse a partir de las mutaciones micro que se producen en contextos de alta inestabilidad, porque en ellos los mecanismos en operación se "ven" con mayor claridad en la medida en que los efectos sobre las variables son más marcados que en situaciones de desequilibrio leve.

$\mathrm{Al}$ analizar las influencias de lo macro sobre la estructura micro, una conclusión central fue la de que las mutaciones micro inducidas por la inestabilidad macroeconomica tienen la particularidad de afectar el grado de preferencia de los agentes por la flexibilidad. En relación con los problemas de crecimiento antes enumerados, los cambios en el grado de preferencia por la flexibilidad son fundamentales, porque inducen variaciones no sólo en la cuantía sino también en la eficiencia de la inversión, así como en la capacidad de innovación.

En una situación de alta preferencia por la flexibilidad, el acortamiento del horizonte temporal hace que los proyectos de inversión con períodos de maduración corto tengan un premio económico sobre los de mayor duración $y$, a su vez, que a igual período de maduración, los beneficios esperados de los proyectos con mayor riesgo sean descontados utilizando una tasa excesivamente alta. Asimismo, se observa empíricamente que las correcciones en las tasas de descuento para proyectos largos o con mayor riesgo en contextos altamente inestables no sólo incorporan rápidamente la información sobre los aumentos del riesgo sistémico sino que, en realidad, tienden a sobrerreaccionar. A su vez, los fenómenos de sobrerreacción inducen a marcados errores en la asignación intertemporal de recursos, al sesgar las señales de precio y cantidad. De esto se sigue que la inversión tenderá a ser ineficiente y que esto agravará el problema neoclásico.

En general, en una situación de alta preferencia por la flexibilidad, se privilegiarán los proyectos de inversión de corto plazo que muestran una menor correlación con el ciclo económico y una menor dispersión de rendimientos. Al aumentar la preferencia por la flexibilidad en América Latina en los años ochenta, por ejemplo, sólo una reducida cantidad de proyectos de inversión podían cumplir con las condiciones señaladas; no es sorprendente entonces que haya habido abruptas caídas de las tasas de inversion/producto en la mayor parte de los países de la región. $Y$ tampoco sorprende que la inversión extranjera directa se haya orientado a la adquisición de empresas de servicios públicos privatizados, que cumplen con el 
requisito fundamental de ser negocios que manejan una enorme cantidad de recursos líquidos, utilizan tecnologías probadas y se catalogan como inversiones de bajo riesgo.

Por ejemplo, en el libro de las "betas"10 de activos que se utilizan en las bolsas desarrolladas para medir el riesgo económico de los proyectos de inversión, los negocios relacionados con servicios públicos como gas, electricidad y teléfonos son los que figuran con las betas más bajas en la lista (Brealey y Myers, 1986). Esto indica que se trata de negocios de bajo riesgo y con beneficios que no tienden a moverse con las tendencias globales del mercado. Los negocios asociados con la tecnología de vanguardia y la innovación - la electrónica, por ejemplo-, que justamente son los que podrían aportar más en términos de aprendizaje tecnologico en los países de la región, son los que figuran con las betas más altas. Como una beta más alta implica mayor riesgo, en un contexto de alta incertidumbre y caracterizado por la sobrerreacción en la corrección de las tasas de descuento, la rentabilidad requerida para llevar adelante tales proyectos es tan alta que la probabilidad de que un proyecto de tecnología avanzada se lleve a la práctica se reduce a un mínimo. Un comentario adicional: si se tiene en cuenta que la inversión extranjera directa es uno de los vehículos fundamentales de transferencia tecnológica para un país en desarrollo (Dahlman, 1993; Dahlman y Nelson, 1993) y que las privatizaciones abrieron la posibilidad para los inversores extranjeros de adquirir negocios de bajo riesgo, se llega a la conclusión de que en el actual contexto poco puede esperarse de la innovación como fuente del crecimiento. La reforma estructural, en una situación de inestabilidad, puede estar actuando en cierta medida en contra de una mayor sofisticación de la estructura productiva, si la adquisición de empresas públicas desplaza los proyectos de mayor riesgo y sofisticación tecnológica.

En realidad, la actividad de innovación $-\mathrm{y}$ por ende la posibilidad de resolver el problema schumpeteriano- es una de las más profundamente afectadas por los aumentos en la preferencia por la flexibilidad. Esto ocurre en gran medida porque en los países subdesarrollados el aprendizaje tecnológico está íntimamente relacionado con la adquisición de nuevos equipos de producción, de modo que cuando la inversión

\footnotetext{
10 Beta: sensibilidad del retomo del activo respecto de las variacio nes en el conjunto de los retornos de los activos transados en el mercado.
}

cae el proceso de cambio tecnológico se resiente (Bradford, 1994). Hay una segunda razón por la cual el proceso de innovación tiende a paralizarse. Cuando la incertidumbre que acompaña la información de precios, financiera y de cantidades es grande, los empresarios encargados de invertir generalmente adoptan estrategias defensivas. Y las estrategias defensivas debilitan la agresividad competitiva y la disposición a innovar en el proceso productivo (Katz, 1993). En un sentido muy concreto, puede decirse que la preferencia por la flexibilidad, así como tiende a destruir la eficiencia en la operación de los mercados, empeorando el problema neoclásico, también tiende a desintegrar los sistemas de innovación nacionales, al afectar severamente los comportamientos de los agentes y las instituciones que contribuyen a sostenerlos y esto, sin duda hace más severa la restricción schumpeteriana del crecimiento.

Las mutaciones microeconómicas que agudizan la preferencia por la flexibilidad afectan también, y muy significativamente, la estructura de intermediación financiera. En una situación de preferencia generalizada por la flexibilidad en las decisiones microeconómicas, se produce un movimiento generalizado de la demanda de activos financieros hacia el corto plazo y, frecuentemente, también hacia los valores denominados en moneda extranjera (como en Argentina, Uruguay, Perú y Bolivia). Esto significa que los valores líquidos o de muy corto plazo aumentan en relación con los de más larga maduración, y que la relación precio de mercado/valor de emisión de los instrumentos denominados en moneda nacional tenderá a deprimirse. Asimismo, la liquidez pasa a ser un atributo tan importante como el rendimiento a la hora de definir la preferencia por un valor determinado. Esto a su vez tiene como correlato, por el lado de la generación de crédito, que las condiciones para acceder a fondos de largo plazo son cada vez más duras y que, cuando el crédito disponible lo es en moneda extranjera, las empresas ligadas en su actividad al mercado interno estarán obligadas a asumir un riesgo cambiario mayor.

Dicho en otros términos, al salto de las tasas de descuento debido al riesgo económico del negocio que hemos analizado más arriba, hay que agregar el aumento en tales tasas debido al salto en los riesgos financieros. Así, dos proyectos de inversión con igual riesgo económico pero que muestran diferente riesgo financiero - porque la estructura de financiamiento a la cual puede accederse para financiar uno y otro es diferente- pueden no obtener la misma calificación 
en el proceso de selección. Podría ocurrir que uno se lleve a cabo y otro no. Es un hecho conocido que en cualquier economía el riesgo financiero afecta al riesgo económico de un negocio. La diferencia específica de una economía que muestra una excesiva preferencia por la flexibilidad es que tal diferencia se exacerba.

Obviamente, esto determina en gran medida la capacidad de crecimiento, al afectar el proceso por el cual se da la selección de los ganadores en la pugna competitiva. La selección pasa a estar muy fuertemente relacionada con las características financieras específicas de cada rama de la producción, y mucho menos con las características económicas. Luego de un período prolongado, en una economía con mercados de capital de largo plazo faltantes o demasiado estrechos, las firmas que sobreviven en el proceso no son necesariamente aquéllas con mayor capacidad para asignar eficientemente los recursos existentes o para innovar en productos y procesos productivos, sino las que se encuentran en ramas de la producción que $\rightarrow$ por el tipo de organización industrial que las caracteriza- se ven menos afectadas por esa falla de mercado.

Las firmas que tienden a sobrevivir mejor en este tipo de ambiente financiero son las que hacen uso intensivo de capital y tienen ingresos previsibles. ${ }^{11}$ Este tipo de empresas pueden aumentar relativamente más su coeficiente de endeudamiento sin producir grandes saltos en sus índices de riesgo financiero. Nuevamente, las ramas de producción que suelen estar más pobladas por este tipo de empresas son las asociadas a los servicios públicos privatizados. En la escala de capacidad de sobrevivencia le siguen las empresas grandes, también con alta densidad de capital, pero con ingresos operativos menos previsibles. En este caso, la capacidad de aumentar el coeficiente de endeudamiento $\sin$ generar saltos en el coeficiente de riesgo financiero es menor (los ejemplos típicos son las firmas que actúan en ramas como el acero, el cemento y la petroquímica). Por último, las empresas que muestran una mayor vulnerabilidad son las medianas y pequeñas cuyos ingresos operativos se relacionan fuertemente con el ciclo económico global, como las que suelen pulular en amplias franjas del sector industrial latinoamericano (rama textil, pequenos productores de bienes de capital, etc.). Al sesgar las bases de selección "natural" en el proceso competitivo, se establecen fuerzas que actúan en contra de

\footnotetext{
1 Vease una categorización de las características financieras del financiamiento empresarial en función de las características de la rama de producción, en Banco Mundial, 1989.
}

la solución del problema tanto neoclásico como schumpeteriano.

Cabe acotar, asimismo, que la falta de desarrollo del sistema financiero también afecta a tales problemas. Por un lado, la segmentación típica de los mercados de poco desarrollo tiende a perjudicar fuertemente el acceso al crédito de las pequeñas y medianas empresas, reforzando las tendencias antes señaladas en favor de las empresas grandes. Por otro, el sistema bancario contribuye de manera importante a mejorar la asignación de recursos mediante los mecanismos de selección utilizados por los oficiales de crédito en la asignación de préstamos al sector privado. ${ }^{12}$ Cuando el crédito es inexistente, tales mecanismos dejan de funcionar y los inversores privados pierden una fuente importante de evaluación independiente de sus proyectos.

En realidad, algunas de las principales características de las estructuras productivas emanadas de una década de inestabilidad y astringencia fínanciera en América Latina, parecen guardar relación con el "ranking de probabilidad de subsistencia por razones financieras" que acabamos de comentar. En efecto, la interacción de los fenómenos microeconómicos y macroeconómicos en el marco de la crisis de la deuda tuvo como resultado neto el estancamiento económico y la caída de la tasa de inversión. Esto último, unido a la marcada inestabilidad, determinó un singular retroceso en el proceso de diversificación productiva de América Latina. La posibilidad de que se produjera un desarrollo integrado del sector industrial a partir de la experiencia de sustitución de importaciones abortó definitivamente. Los sectores industriales que sobrellevaron la crisis en mejores condiciones fueron básicamente los de procesamiento de recursos naturales y algunos de producción a escala como el automotriz —que de cualquier forma está pasando por un profundo proceso de reconversión con resultado incierto en algunos países. Otros algo más sofisticados, como el de producción de bienes de capital, han tendido a desaparecer y lo mismo ha ocurrido con algunos segmentos del rubro textil. Los sectores dinámicos "nuevos" están asociados con las privatizaciones en países como Chile, Argentina y, en menor medida, México (Guerrieri, 1993; Katz, 1993).

Para finalizar con el tema del crecimiento queremos examinar una última consecuencia importante de las mutaciones micro. Como ya dijimos, las situacio-

\footnotetext{
12 Sobre este rol del sistema financiero, véase Stiglitz, 1993.
} 
nes de preferencia por la flexibilidad suelen traducirse en un fuerte aumento de la demanda de activos en moneda extranjera. En América Latina este fenomeno se traduce en una más profunda dolarización del sistema financiero intemo, o bien en fuga de capitales. Ambos fenómenos agudizan la restricción keynesiana, al dificultar el proceso de intermediación entre el ahorro y la inversión. Sin embargo, las consecuencias de ambos fenómenos no son las mismas. La dolarización, como principal consecuencia adversa, induce a las empresas a tomar un mayor tiesgo cambiario; pero tiene también un rasgo positivo: aumenta la capacidad de prestar del sistema financiero interno (en dólares). Por lo tanto, no implica que el ahorro interno no pueda intermediarse hacia la inversión, sino que los costos de tal intermediación pueden tornarse más caros para las firmas, al hacerlas incunir en un mayor riesgo cambiario.

Es justamente este elemento el que diferencia la dolarización de la fuga de capitales. En el caso de esta última, el aumento de la demanda de activos denominados en moneda extranjera implica que parte del ahorro interno irá a financiar proyectos de inversion en otras economías. Esto tiene consecuencias muy negativas sobre la restriccion keynesiana, porque el proceso de intermediación entre el ahorro y la inversión a nivel interno se interrumpe y el crecimiento -si no se tiene acceso al financiamiento externo, como ocurrí en los años ochentacomienza a estar restringido por la disponibilidad de ahorro. Una forma típica en que esto se manifiesta es a través del racionamiento del crédito bancario. De tal forma, los proyectos de inversión no encuentran fondos que los financien. Y ello no ocurre por- que se ahorre poco internamente, sino porque el ahorro se "exporta" a través de la decisión de cartera de los agentes.

En realidad, históricamente, el desafío impuesto por la restricción keynesiana del crecimiento ha sido uno de los más difíciles de superar en América Latina. La creación de bancos públicos con propósitos de fomento del desarrollo en la región puede interpretarse como un intento de reparar esta falla congénita de la organización económica. Pero, en general, el funcionamiento de tales bancos también adoleció de importantes fallas, que se hicieron críticas a medida que la inestabilidad macroeconómica amplió la magnitud de la distorsiones y aument 6 los incentivos para actividades de persecución de rentas. Esto parece claro en muchos países latinoamericanos -Argentina, Brasil y Chile, entre otros- en los cuales el deterioro de las funciones de canalización de ahorros y de selección y asignación eficiente del crédito estuvo asociado con el incremento de la inflación.

Asimismo, la mayor importancia relativa del ahorro público y el financiamiento de la inversión privada mediante transferencias fiscales también han sido en América Latina la contracara de la debilidad de los sistemas financieros. Pero la crisis fiscal y las necesidades de la estabilización han puesto límites estrictos a ese papel tradicionalmente desempeñado por el sector público. En realidad, la falta de éxito en el desarrollo de estos mecanismos institucionales para suplir la falla de mercado representada por la ausencia de un mercado de capitales de largo plazo, no hace más que probar que la inestabilidad macroeconómica es tan perjudicial para el desamollo de los mercados como para el de las instituciones.

\section{Bibliografia}

Banco Mundial (1989): Informe sobre el desarrollo mundial 1989, Washington, D.C.

Benassy, Jean-Pascal (1982): The Economics of Market Disequilibrium, Nueva York, Academic Press.

Bradford, Colin I. (1994): The new paradigm of systemic competitiveness: Why it matters, what it means and implications for policy, París, Organización de Cooperación y Desarrollo Econónicos (OCDE), mimeo.

Brealey, R. y S. Myers (1986): Principtos de finanzas corporativas, Buenos Aires, Mc Graw Hill.

Dahlman, Carl J. (1993): New elements of international competitiveness: Implications for technology policy in developing economies, Washington, D.C., Banco Mundial, mimeo.

Dahlman, C. y R. Nelson (1993): Social absorption capability, national innovation systems and economic development, Maastricht, Paises Bajos, Universidad de las Naciones Unidas (UNU), Instituto de Nuevas Tecnologias (INTECH).
Damill, M., J. M. Fanelli, R. Frenkel y G. Rozenwurcel (1993): Crecimiento económico en América Latina: experiencia reciente y perspectivas, Desarrollo económico, vol. $33, \mathrm{~N}^{\circ} 130$, Buenos Aires, Instituto de Desarrollo Económico y Social, julio-setiembre.

Fanelli, José M. (1988): Desequilibrio macroeconómico, restricciones financieras y políticas de estabilizacion, tesis de doctorado, mimeo.

Faneilli, J., R. Frenkel y L. Taylor (1992): The World Development Report 1991: A critical assessment, Naciones Unidas, International Monetary and Financial lssues for the 1990s, vol. I, Nueva York.

Fanelli, J. y R. Frenkel (1994): Macropolicies for the Transition from Stabilization to Growth, Documento Cedes, $N^{\circ} 87$, Buenos Aires, Centro de Estudios de Estado y Sociedad (CEDEs).

Frenkel, R. (1990): El régimen de alta inflación y el nivel de actividad, J. P. Arellano (comp.), Infiacion rebelde en Amé- 
rica Latina, Santiago de Chile, Corporación de Investigaciones Económicas para América Latina (CIEPLAN)/Hachette.

Guerrieri, Paolo (1993); Technological interdependence and international competitiveness in major Latin American countries, trabajo presentado a la Conference on Integrating Competitiveness, Sustainability and Social Development, Santiago de Chile, mimeo.

Hicks, John (1974); The Crisis in Keynesian Economics, Oxford, Inglaterra, Basil Blackwel.

Johnson B. y B. Lundvall (1988): Institutional leaming and national systems of innovation, trabajo presentado en la conferencia Strategies of Flexibilisation in Europe, Dinamarca, Roskilde Universitetscenter, mimeo.

Katz, Jorge (1993): Texhnology and industrial re-estructuring in Latin America: Emerging new issues of the 1990's, Buenos Aires, Comisión Econónica para América Latina y el Caribe (CEPAL).

Leijonhufvud, Axel (1968): On the Keynesian Economics and the
Economics of Keynes. A Study in Monetary Theory, Nueva York, Oxford University Press.

Mowery, David C. (1993): Inward technology transfer and competitiveness: The role of national innovation systems, trabajo presentado en la UNU/WNECH Research Conference, Maastricht, Países Bajos.

Nelson, Richard R. (1991): Why do firms differ, and how does it matter?, Strategic Management Journal, vol. 12.

_. (1992): Schumpeter, and contemporary research on the economics of innovation, Nueva York, Universidad de Columbia, mimeo.

(ed.) (1993): National Innovation Systems. A Comparative Analysis, Nueva York, Oxford University Press.

Scherer, F. (1980): Industrial Market Structure and Economic Performance, Chicago, Ill., Estados Unidos, Rand-McNally, segunda edición.

Stiglitz, Joseph E. (1993): The role of the State in financial markets, trabajo presentado en la Annual Bank Conference on Development Economics. Washington, D.C., Banco Mundial. 